\title{
Pacific
}

Journal of

Mathematics

\section{TWISTED SYMMETRIC GROUP ACTIONS}

AKINARI Hoshi AND MING-CHANG KANG 


\title{
TWISTED SYMMETRIC GROUP ACTIONS
}

\author{
AKINARI HOSHI AND MING-CHANG KANG
}

Let $K$ be any field, let $K\left(x_{1}, \ldots, x_{n}\right)$ be the rational function field of $n$ variables over $K$, and let $S_{n}$ and $A_{n}$ be the symmetric group and the alternating group of degree $n$, respectively. For any $a \in K \backslash\{0\}$, define an action of $S_{n}$ on $K\left(x_{1}, \ldots, x_{n}\right)$ by $\sigma \cdot x_{i}=x_{\sigma(i)}$ for $\sigma \in A_{n}$ and $\sigma \cdot x_{i}=a / x_{\sigma(i)}$ for $\sigma \in S_{n} \backslash A_{n}$. We prove that for any field $K$ and $n=3,4,5$, the fixed field $K\left(x_{1}, \ldots, x_{n}\right)^{S_{n}}$ is rational (that is, purely transcendental) over $K$.

\section{Introduction}

Let $K$ be any field, let $K\left(x_{1}, \ldots, x_{n}\right)$ be the rational function field of $n$ variables over $K$, and let $S_{n}$ and $A_{n}$ be the symmetric group and the alternating group of degree $n$, respectively. For any $a \in K \backslash\{0\}$, define a twisted action of $S_{n}$ on $K\left(x_{1}, \ldots, x_{n}\right)$ by

$$
\sigma\left(x_{i}\right):= \begin{cases}x_{\sigma(i)} & \text { if } \sigma \in A_{n}, \\ a / x_{\sigma(i)} & \text { if } \sigma \in S_{n} \backslash A_{n} .\end{cases}
$$

Consider the fixed subfield

$$
K\left(x_{1}, \ldots, x_{n}\right)^{S_{n}}=\left\{\alpha \in K\left(x_{1}, \ldots, x_{n}\right): \sigma(\alpha)=\alpha \text { for any } \sigma \in S_{n}\right\} .
$$

If $n=2$, then $K\left(x_{1}, x_{2}\right)^{S_{2}}=K\left(x_{1}+\left(a / x_{2}\right), a x_{1} / x_{2}\right)$ is rational (that is, purely transcendental) over $K$. When $a=1$ (equivalently when $a \in K^{\times 2}$ ), we have the following theorem.

Theorem 1.1 [Hajja and Kang 1997, Theorem 3.5]. Let $K$ be any field and let $a \in K^{\times 2}$. Then $K\left(x_{1}, \ldots, x_{n}\right)^{S_{n}}$ is rational over $K$.

The case when $a \in K^{\times} \backslash K^{\times 2}$ and $n \geq 3$ had been intractable for many years; see [Hajja and Kang 1997, page 638; Hajja 2000, Example 5.12, page 147; Kang 2001, Question 3.8, page 215]. Even the case $n=3$ was unsolved. The next theorem is our recent result for the cases $n=3,4,5$.

MSC2000: 13A50, 12F20.

Keywords: rationality problem, conic bundles.

Hoshi was partially supported by Waseda University Grant for Special Research Projects number 2007B-067 and Rikkyo University Special Fund for Research. Kang was partially supported by National Center for Theoretic Sciences, Taipei Office. 
Theorem 1.2. Let $K$ be any field, let $a \in K \backslash\{0\}$, and let $S_{n}$ act on $K\left(x_{1}, \ldots, x_{n}\right)$ as defined in (1-1). If $n=3,4,5$, then $K\left(x_{1}, \ldots, x_{n}\right)^{S_{n}}$ is rational over $K$.

We will prove Theorem 1.2 in Section 2. It is interesting that we use three different methods for the three cases of $n$; it seems that there is no unified proof for the three cases. One of the reasons is that the solutions to Noether's problem for the alternating group $A_{n}$ are rather different when $n=3$ and when $n=5$; see Theorem 2.2 and Theorem 2.5. Since Noether's problem for $A_{n}$ is still open in the case $n \geq 6$ (see [Maeda 1989] and [Hajja and Kang 1995, Section 4] for the statement of this problem), it is not so surprising that our question is solvable at present only for $n \leq 5$. It is still unknown whether the fixed field $K\left(x_{1}, \ldots, x_{n}\right)^{S_{n}}$ is rational when $n \geq 6$.

In Section 3 we propose another approach to the rationality of $K\left(x_{1}, \ldots, x_{n}\right)^{S_{n}}$. We show in Theorem 3.4 that it is isomorphic to the function field of a conic bundle over $\mathbb{P}^{n-1}$ of the form $x^{2}-a y^{2}=h\left(v_{1}, \ldots, v_{n-1}\right)$ with affine coordinates $v_{1}, \ldots, v_{n-1}$. Although this approach is valid only when char $K \neq 2$, it does provide a new technique in studying rationality problems. The structure of a conic bundle together with its rationality problem is a central subject in algebraic geometry [Iskovskih 1991]. Fortunately, when $n=3$ and $n=4$, the conic bundle in our case contains singularities and the rationality problem can be solved by a suitable blowing-up process. In particular, we find another proof of Theorem 1.2 when char $K \neq 2$ and $n=3$, 4. For other rationality problems of conic bundles, see [Kang 2007, Section 4].

Since the fixed field $K\left(x_{1}, \ldots, x_{n}\right)^{S_{n}}$ is the quotient field of the ring of invariants $K\left[x_{1}, \ldots, x_{n}\right]^{S_{n}}$, it seems plausible to study it through the structure of the latter. This strategy is carried out in Section 4, and we give another proof of Theorem 1.2 when $\operatorname{char} K=2$ and $n=3,4$.

\section{Proof of Theorem 1.2}

Theorem 2.1 [Kang 2004, Theorem 2.4]. Let $K$ be any field and let $K(x, y)$ be the rational function field of two variables over $K$. Let $\sigma$ be a $K$-automorphism on $K(x, y)$ defined by

$$
\sigma: x \mapsto a / x, \quad y \mapsto b / y,
$$

where $a \in K \backslash\{0\}$ and $b=c(x+(a / x))+d$ such that $c, d \in K$ and at least one of $c$ and $d$ is nonzero. Then $K(x, y)^{\langle\sigma\rangle}=K(s, t)$, where

$$
s=\frac{x-(a / x)}{x y-(a b / x y)}, \quad t=\frac{y-(b / y)}{x y-(a b / x y)} .
$$

The next result is essentially due to Masuda [1955, page 62] when char $K \neq 3$ (with a misprint in the original expression). We thank Y. Rikuna who pointed out 
that the same formula is still valid when char $K=3$ if we compare this formula with the proof in [Kuniyoshi 1955]. For convenience, we provide a new proof.

Theorem 2.2 [Masuda 1955, Theorem 3]. Let $K$ be any field, $K\left(x_{1}, x_{2}, x_{3}\right)$ be the rational function field of three variables over $K$. Let $\sigma$ be a $K$-automorphism on $K\left(x_{1}, x_{2}, x_{3}\right)$ defined by

$$
\sigma: x_{1} \mapsto x_{2} \mapsto x_{3} \mapsto x_{1}
$$

Then $K\left(x_{1}, x_{2}, x_{3}\right)^{\langle\sigma\rangle}=K\left(s_{1}, u, v\right)=K\left(s_{3}, u, v\right)$, where $s_{i}$ is the elementary symmetric function of degree $i$ for $1 \leq i \leq 3$, and $u$ and $v$ are defined by

$$
\begin{aligned}
u & :=\frac{x_{1} x_{2}^{2}+x_{2} x_{3}^{2}+x_{3} x_{1}^{2}-3 x_{1} x_{2} x_{3}}{x_{1}^{2}+x_{2}^{2}+x_{3}^{2}-x_{1} x_{2}-x_{2} x_{3}-x_{3} x_{1}}, \\
v & :=\frac{x_{1}^{2} x_{2}+x_{2}^{2} x_{3}+x_{3}^{2} x_{1}-3 x_{1} x_{2} x_{3}}{x_{1}^{2}+x_{2}^{2}+x_{3}^{2}-x_{1} x_{2}-x_{2} x_{3}-x_{3} x_{1}} .
\end{aligned}
$$

Moreover, we have the identities

$$
\begin{aligned}
s_{2} & =s_{1}(u+v)-3\left(u^{2}-u v+v^{2}\right), \\
s_{3} & =s_{1} u v-\left(u^{3}+v^{3}\right), \\
x_{1} x_{2}^{2}+x_{2} x_{3}^{2}+x_{3} x_{1}^{2} & =s_{1}^{2} u-3 s_{1} u^{2}+3(2 u-v)\left(u^{2}-u v+v^{2}\right), \\
x_{1}^{2} x_{2}+x_{2}^{2} x_{3}+x_{3}^{2} x_{1} & =s_{1}^{2} v-3 s_{1} v^{2}-3(u-2 v)\left(u^{2}-u v+v^{2}\right) .
\end{aligned}
$$

Proof. With the aid of computer packages, say Mathematica or Maple, it is easy to verify the theorem's identities. We have $\left[K\left(x_{1}, x_{2}, x_{3}\right): K\left(s_{1}, s_{2}, s_{3}\right)\right]=6$ and $\left[K\left(x_{1}, x_{2}, x_{3}\right)^{\langle\sigma\rangle}: K\left(s_{1}, s_{2}, s_{3}\right)\right]=2$. Since $x_{1} x_{2}^{2}+x_{2} x_{3}^{2}+x_{3} x_{1}^{2} \notin K\left(s_{1}, s_{2}, s_{3}\right)$, it follows that $K\left(x_{1}, x_{2}, x_{3}\right)^{\langle\sigma\rangle}=K\left(s_{1}, s_{2}, s_{3}, x_{1} x_{2}^{2}+x_{2} x_{3}^{2}+x_{3} x_{1}^{2}\right) \subset K\left(s_{1}, u, v\right)$. Hence $K\left(x_{1}, x_{2}, x_{3}\right)^{\langle\sigma\rangle}=K\left(s_{1}, u, v\right)=K\left(s_{3}, u, v\right)$.

Proof of Theorem 1.2 when $n=3$. Let $\sigma=(1,2,3), \tau=(1,2) \in S_{3}$.

By Theorem 2.2, we find that $K\left(x_{1}, x_{2}, x_{3}\right)^{\langle\sigma\rangle}=K\left(s_{3}, u, v\right)$.

Now $\tau\left(x_{1}\right)=a / x_{2}, \tau\left(x_{2}\right)=a / x_{3}$, and $\tau\left(x_{3}\right)=a / x_{3}$. Note that

$$
\begin{aligned}
& \tau\left(s_{1}\right)=a s_{2} / s_{3}, \quad \tau\left(s_{2}\right)=a^{2} s_{1} / s_{3}, \quad \tau\left(s_{3}\right)=a^{3} / s_{3}, \\
& \tau\left(x_{1} x_{2}^{2}+x_{2} x_{3}^{2}+x_{3} x_{1}^{2}\right)=a^{3}\left(x_{1} x_{2}^{2}+x_{2} x_{3}^{2}+x_{3} x_{1}^{2}\right) / s_{3}^{2}, \\
& \tau\left(x_{1}^{2} x_{2}+x_{2}^{2} x_{3}+x_{3}^{2} x_{1}\right)=a^{3}\left(x_{1}^{2} x_{2}+x_{2}^{2} x_{3}+x_{3}^{2} x_{1}\right) / s_{3}^{2} .
\end{aligned}
$$

With the aid of Theorem 2.2, it is not difficult to find that

$$
\tau: s_{3} \mapsto \frac{a^{3}}{s_{3}}, \quad u \mapsto \frac{a u}{u^{2}-u v+v^{2}}, \quad v \mapsto \frac{a v}{u^{2}-u v+v^{2}} .
$$


Define $w:=u / v$. Then $K\left(s_{3}, u, v\right)=K\left(s_{3}, v, w\right)$ and

$$
\tau: s_{3} \mapsto \frac{a^{3}}{s_{3}}, \quad v \mapsto \frac{a}{v\left(1-w+w^{2}\right)}, \quad w \mapsto w .
$$

By Theorem 2.1, $K\left(s_{3}, v, w\right)^{\langle\tau\rangle}$ is rational over $K(w)$. Hence $K\left(x_{1}, x_{2}, x_{3}\right)^{S_{3}}=$ $K\left(s_{3}, v, w\right)^{\langle\tau\rangle}$ is rational over $K$.

Proof of Theorem 1.2 when $n=4$. Define

$$
\begin{array}{rllll}
\sigma & :=(123) \quad: x_{1} \mapsto x_{2} \mapsto x_{3} \mapsto x_{1}, \\
\tau & :=(12) \quad: x_{1} \mapsto a / x_{2}, \quad x_{2} \mapsto a / x_{1}, & x_{3} \mapsto a / x_{3}, & x_{4} \mapsto a / x_{4}, \\
\rho_{1}: & :=(12)(34): x_{1} \mapsto x_{2}, \quad x_{2} \mapsto x_{1}, & x_{3} \mapsto x_{4}, & x_{4} \mapsto x_{3}, \\
\rho_{2} & :=(13)(24): x_{1} \mapsto x_{3}, \quad x_{3} \mapsto x_{1}, & x_{2} \mapsto x_{4}, & x_{4} \mapsto x_{2} .
\end{array}
$$

Note that $\{1\} \triangleleft V_{4}=\left\langle\rho_{1}, \rho_{2}\right\rangle \triangleleft A_{4}=\left\langle\sigma, \rho_{1}, \rho_{2}\right\rangle \triangleleft S_{4}=\left\langle\sigma, \tau, \rho_{1}, \rho_{2}\right\rangle$ is a normal series.

First we will show that $K\left(x_{1}, \ldots, x_{4}\right)^{V_{4}}$ is rational over $K$. Define

$$
\begin{aligned}
s_{1} & :=x_{1}+x_{2}+x_{3}+x_{4}, \quad s_{4}:=x_{1} x_{2} x_{3} x_{4}, \\
S & :=\frac{x_{1}+x_{2}-x_{3}-x_{4}}{x_{1} x_{2}-x_{3} x_{4}}, \quad T:=\frac{x_{1}-x_{2}-x_{3}+x_{4}}{x_{1} x_{4}-x_{2} x_{3}}, \quad U:=\frac{x_{1}-x_{2}+x_{3}-x_{4}}{x_{1} x_{3}-x_{2} x_{4}} .
\end{aligned}
$$

Then we have $K\left(s_{1}, s_{4}, S, T, U\right) \subset K\left(x_{1}, x_{2}, x_{3}, x_{4}\right)^{V_{4}}$ and

$$
\sigma: s_{1} \mapsto s_{1}, \quad s_{4} \mapsto s_{4}, \quad S \mapsto T, \quad T \mapsto U, \quad U \mapsto S .
$$

Lemma 2.3. (i) $K\left(x_{1}, x_{2}, x_{3}, x_{4}\right)^{V_{4}}=K\left(s_{1}, S, T, U\right)=K\left(s_{4}, S, T, U\right)$.

(ii) $K\left(x_{1}, x_{2}, x_{3}, x_{4}\right)^{A_{4}}=K\left(s_{4}, f, g, h\right)$ where $f, g$, $h$ are defined by

$$
\begin{aligned}
f=S+T+U, \quad g & =\frac{S T^{2}+T U^{2}+U S^{2}-3 S T U}{S^{2}+T^{2}+U^{2}-S T-T U-U S}, \\
h & =\frac{S^{2} T+T^{2} U+U^{2} S-3 S T U}{S^{2}+T^{2}+U^{2}-S T-T U-U S} .
\end{aligned}
$$

Proof. Define $u_{1}:=S+T+U, u_{2}:=S T+T U+S U$ and $u_{3}:=S T U$. Then it can be checked that $K\left(x_{1}, x_{2}, x_{3}, x_{4}\right)=K\left(s_{1}, S, T, U\right)\left(x_{4}\right)$ directly from the equalities

$$
\begin{aligned}
& x_{1}=\frac{4-s_{1} T+\left(-2 u_{1}+s_{1} T(S+U)\right) x_{4}+S U\left(1-s_{1} T\right) x_{4}^{2}+u_{3} x_{4}^{3}}{S-T+U-S U x_{4}}, \\
& x_{2}=\frac{4-s_{1} U+\left(-2 u_{1}+s_{1} U(T+S)\right) x_{4}+T S\left(1-s_{1} U\right) x_{4}^{2}+u_{3} x_{4}^{3}}{T-U+S-T S x_{4}}, \\
& x_{3}=\frac{4-s_{1} S+\left(-2 u_{1}+s_{1} S(U+T)\right) x_{4}+U T\left(1-s_{1} S\right) x_{4}^{2}+u_{3} x_{4}^{3}}{U-S+T-U T x_{4}} .
\end{aligned}
$$


We see that $\left[K\left(s_{1}, S, T, U\right)\left(x_{4}\right): K\left(s_{1}, S, T, U\right)\right] \leq 4$ by the equality

$$
u_{1}^{2}-4 u_{2}+s_{1} u_{3}+\left(8-s_{1} u_{1}\right) u_{3} x_{4}-\left(2 u_{1}-s_{1} u_{2}\right) u_{3} x_{4}^{2}-s_{1} u_{3}^{2} x_{4}^{3}+u_{3}^{2} x_{4}^{4}=0 .
$$

Hence we get $K\left(x_{1}, x_{2}, x_{3}, x_{4}\right)^{V_{4}}=K\left(s_{1}, S, T, U\right)$. It follows from the equality $s_{4}=\left(u_{1}^{2}-4 u_{2}+u_{3} s_{1}\right) / u_{3}^{2}$ that $K\left(s_{1}, S, T, U\right)=K\left(s_{4}, S, T, U\right)$.

As for the field $K\left(x_{1}, x_{2}, x_{3}, x_{4}\right)^{A_{4}}$, apply Theorem 2.2 to $K\left(s_{4}, S, T, U\right)^{\langle\sigma\rangle}$ $=K(S, T, U)^{\langle\sigma\rangle}\left(s_{4}\right)$.

We have $K\left(x_{1}, x_{2}, x_{3}, x_{4}\right)^{S_{4}}=\left(K\left(x_{1}, x_{2}, x_{3}, x_{4}\right)^{V_{4}}\right)^{S_{4} / V_{4}}=K\left(s_{4}, S, T, U\right)^{\langle\sigma, \tau\rangle}$. The action of $\langle\sigma, \tau\rangle$ on $K\left(s_{4}, S, T, U\right)$ is given by

$$
\begin{aligned}
& \sigma: s_{4} \mapsto s_{4}, \quad S \mapsto T, \quad T \mapsto U, \quad U \mapsto S, \\
& \tau: s_{4} \mapsto \frac{a^{4}}{s_{4}}, \quad S \mapsto \frac{-S+T+U}{a T U}, \quad T \mapsto \frac{S+T-U}{a S T}, \quad U \mapsto \frac{S-T+U}{a S U} .
\end{aligned}
$$

Define

$$
N:= \begin{cases}\frac{s_{4}+a^{2}}{s_{4}-a^{2}} & \text { if char } K \neq 2 \\ \frac{s_{4}}{s_{4}+a^{2}} & \text { if char } K=2 .\end{cases}
$$

Then we get $K\left(s_{4}, S, T, U\right)=K(N, S, T, U), \sigma(N)=N$ and

$$
\tau(N)= \begin{cases}-N & \text { if } \operatorname{char} K \neq 2 \\ N+1 & \text { if } \operatorname{char} K=2\end{cases}
$$

Applying [Hajja and Kang 1995, Theorem 1], we find that $K\left(x_{1}, x_{2}, x_{3}, x_{4}\right)^{S_{4}}=$ $K(N, S, T, U)^{\langle\sigma, \tau\rangle}$ is rational over $K$, provided that $K(S, T, U)^{\langle\sigma, \tau\rangle}$ is rational over $K$. Explicitly, define $P$ by

$$
P:= \begin{cases}N \cdot\left(S+T+U+\frac{S^{2}+T^{2}+U^{2}-2(S T+T U+U S)}{a S T U}\right) & \text { if char } K \neq 2, \\ N+\frac{S+T+U}{S+T+U+a S T U} & \text { if char } K=2 .\end{cases}
$$

Then we have that $K(N, S, T, U)=K(P, S, T, U)$ and $K\left(x_{1}, x_{2}, x_{3}, x_{4}\right)^{S_{4}}=$ $K(P, S, T, U)^{\langle\sigma, \tau\rangle}=K(S, T, U)^{\langle\sigma, \tau\rangle}(P)$, where $\sigma(P)=\tau(P)=P$.

Thus it remains to prove this:

Theorem 2.4. Let $K$ be any field and let $K(S, T, U)$ be the rational function field of three variables $S, T$ and $U$ over $K$. Let $\sigma$ and $\tau$ be $K$-automorphisms of $K(S, T, U)$ defined by

$$
\begin{aligned}
\sigma: S \mapsto T, & T \mapsto U, & U \mapsto S, \\
\tau: S \mapsto \frac{-S+T+U}{a T U}, & T \mapsto \frac{S+T-U}{a S T}, & U \mapsto \frac{S-T+U}{a S U},
\end{aligned}
$$

where $a \in K \backslash\{0\}$. Then $\langle\sigma, \tau\rangle \cong S_{3}$ and $K(S, T, U)^{\langle\sigma, \tau\rangle}$ is rational over $K$. 
Proof. By Theorem 2.2, we may choose a transcendence basis of $K(S, T, U)^{\langle\sigma\rangle}$ over $K$ by $K(S, T, U)^{\langle\sigma\rangle}=K(f, g, h)$, where

$$
\begin{aligned}
f=S+T+U, \quad g & =\frac{S T^{2}+T U^{2}+U S^{2}-3 S T U}{S^{2}+T^{2}+U^{2}-S T-T U-U S}, \\
h & =\frac{S^{2} T+T^{2} U+U^{2} S-3 S T U}{S^{2}+T^{2}+U^{2}-S T-T U-U S} .
\end{aligned}
$$

Thus we have $K(S, T, U)^{\langle\sigma, \tau\rangle}=\left(K(S, T, U)^{\langle\sigma\rangle}\right)^{\langle\tau\rangle}=K(f, g, h)^{\langle\tau\rangle}$. The action of $\tau$ on $K(f, g, h)$ is given by

$$
\begin{aligned}
& f \mapsto \frac{f^{2}-4 f(g+h)+12 X}{a Y}, \\
& g \mapsto \frac{-f^{2} h(f-4 h)+2 f(f-2 g-8 h) X+24 X^{2}-8 g Y}{a\left(f^{2}-2 f(g+h)+4 X\right) Y}, \\
& h \mapsto \frac{-f^{2}\left(f g+4 h^{2}\right)+6 f(f-2 g) X+24 X^{2}-4(f+2 h) Y}{a\left(f^{2}-2 f(g+h)+4 X\right) Y},
\end{aligned}
$$

where $X=g^{2}-g h+h^{2}$ and $Y=g^{3}-f g h+h^{3}$.

Case 1: char $K \neq 2$.

Define

$$
F:=g+h, \quad G:=g-h, \quad H:=f-(g+h) .
$$

Then $K(S, T, U)^{\langle\sigma\rangle}=K(f, g, h)=K(F, G, H)$ and $\tau$ acts on $K(F, G, H)$ by

$$
\begin{gathered}
F \mapsto \frac{4\left(27 G^{4}-7 F G^{2} H+5 G^{2} H^{2}-F H^{3}\right)}{a\left(4 F G^{2}-F^{2} H+G^{2} H\right)\left(3 G^{2}+H^{2}\right)}, \\
G \mapsto \frac{4 G\left(F G^{2}+7 G^{2} H-F H^{2}+H^{3}\right)}{a\left(4 F G^{2}-F^{2} H+G^{2} H\right)\left(3 G^{2}+H^{2}\right)}, \\
H \mapsto \frac{4 H\left(F G^{2}+7 G^{2} H-F H^{2}+H^{3}\right)}{a\left(4 F G^{2}-F^{2} H+G^{2} H\right)\left(3 G^{2}+H^{2}\right)} .
\end{gathered}
$$

Note that $\tau(G / H)=G / H$. Define

$$
A:=F / G, \quad B:=G, \quad C:=G / H .
$$

Then $K(S, T, U)^{\langle\sigma\rangle}=K(F, G, H)=K(A, B, C)$ and $\tau$ acts on $K(A, B, C)$ by

$$
\begin{aligned}
& A \mapsto \frac{-A+5 C-7 A C^{2}+27 C^{3}}{1-A C+7 C^{2}+A C^{3}}, \\
& B \mapsto \frac{4\left(1-A C+7 C^{2}+A C^{3}\right)}{a B\left(1-A^{2}+4 A C\right)\left(1+3 C^{2}\right)}, \quad C \mapsto C .
\end{aligned}
$$

Define

$$
D:=1-A C+7 C^{2}+A C^{3}, \quad E:=2 C\left(C^{2}-1\right) / B .
$$


Then $K(A, B, C)=K(C, D, E)$ and the action of $\tau$ on $K(C, D, E)$ is given by

$$
\begin{aligned}
& C \mapsto C, \quad D \mapsto\left(1+3 C^{2}\right)^{3} / D, \\
& E \mapsto-a\left(1+3 C^{2}\right)\left(D+\left(1+3 C^{2}\right)^{3} / D-2\left(1+5 C^{2}+2 C^{4}\right)\right) / E .
\end{aligned}
$$

Hence the assertion follows from Theorem 2.1.

Case 2: $\operatorname{char} K=2$.

The action of $\tau$ on $K(f, g, h)$ is given by

$$
\tau: f \mapsto \frac{f^{2}}{a Y}, \quad g \mapsto \frac{f h}{a Y}, \quad h \mapsto \frac{f g}{a Y},
$$

where $Y=g^{3}+f g h+h^{3}$. Define

$$
A:=f /(g+h), \quad B:=g / h, \quad C:=1 / h .
$$

Then $K(f, g, h)=K(A, B, C)$ and $\tau$ acts on $K(A, B, C)$ by

$$
A \mapsto A, \quad B \mapsto \frac{1}{B}, \quad C \mapsto \frac{a}{A}\left(B+\frac{1}{B}+A+1\right) / C .
$$

Hence the assertion follows from Theorem 2.1. We will give another proof when $n=4$ and char $K=2$ in Section 4.

This concludes the proof of Theorem 1.2 when $n=4$.

Proof of Theorem 1.2 when $n=5$.

We recall Maeda's theorem for the $A_{5}$ action.

Theorem 2.5 [Maeda 1989]. Let $K$ be any field, $K\left(x_{1}, \ldots, x_{5}\right)$ be the rational function field of five variables over $K$. Then $K\left(x_{1}, \ldots, x_{5}\right)^{A_{5}}$ is rational over $K$. Moreover a transcendental basis $F_{1}, \ldots, F_{5}$ of $K\left(x_{1}, \ldots, x_{5}\right)^{A_{5}}$ over $K$ may be given explicitly as follows:

(i) When char $K \neq 2$,

$$
\begin{aligned}
F_{1} & =\frac{\sum_{\sigma \in S_{5}} \sigma\left([12][13][14][15][23]^{4}[45]^{4} x_{1}\right)}{\sum_{\sigma \in S_{5}} \sigma\left([12][13][14][15][23]^{4}[45]^{4}\right)}, \\
F_{2} & =\frac{\sum_{\sigma \in S_{5}} \sigma\left([12]^{3}[13]^{3}[14]^{3}[15]^{3}[23]^{10}[45]^{10}\right)}{\prod_{i<j}[i j]^{2} \cdot \sum_{\sigma \in S_{5}} \sigma\left([12][13][14][15][23]^{4}[45]^{4}\right)}, \\
F_{3} & =\frac{\sum_{\sigma \in S_{5}} \sigma\left([12]^{3}[13]^{3}[14]^{3}[15]^{3}[23]^{10}[45]^{10} x_{1}\right)}{\prod_{i<j}[i j]^{2} \cdot \sum_{\sigma \in S_{5}} \sigma\left([12][13][14][15][23]^{4}[45]^{4}\right)}, \\
F_{4} & =\frac{\sum_{\mu \in R_{1}} \mu\left([12]^{2}[13]^{2}[23]^{2}[45]^{4}\right)}{\prod_{i<j}[i j]}, \\
F_{5} & =\frac{\sum_{\mu \in R_{1}} \mu\left([12]^{2}[13]^{2}[23]^{2}[14]^{4}[24]^{4}[34]^{4}[15]^{4}[25]^{4}[35]^{4}\right)}{\prod_{i<j}[i j]^{3}},
\end{aligned}
$$


where $[i j]=x_{i}-x_{j}$ and $R_{1}=\{1,(34),(354),(234)$, (2354), (24)(35), (1234), (12354), (124) (35), (13524)\}.

(ii) When $\operatorname{char} K=2$,

$$
\begin{aligned}
& F_{1}=\frac{\sum_{i<j<k} x_{i} x_{j} x_{k}}{\sum_{i<j} x_{i} x_{j}}, \quad F_{4}=\frac{\sum_{v \in R_{3}} v\left([12]^{2}[34]^{2}[13][24][15][25][35][45]\right)}{\prod_{i<j}[i j]}, \\
& F_{2}=\frac{\sum_{i=1}^{5}\left([12][13][14][15] \cdot I^{2}\right)^{(1 i)}}{\prod_{i<j}[i j] \cdot \sum_{i<j} x_{i} x_{j}}, \\
& F_{3}=\frac{\sum_{i=1}^{5}\left([12][13][14][15] \cdot I^{2} \cdot x_{1}\right)^{(1 i)}}{\prod_{i<j}[i j] \cdot \sum_{i<j} x_{i} x_{j}},
\end{aligned}
$$

where $[i j]=x_{i}-x_{j}, I=\sum_{\tau \in R_{2}} \tau\left(x_{2} x_{3}\left(x_{2} x_{3}+x_{4}^{2}+x_{5}^{2}\right)\right), R_{2}=\{1,(34)$, (354), (234), (2354), (24)(35) $\}$ and $R_{3}=\{1$, (234), (243), (152), (15234), (15243), (125), (12345), (12435), (15432), (154), (15423), (15342), (15324), (153)\}.

In the theorem, note that $R_{1}, R_{2}$ and $R_{3}$ are coset representatives with respect to various subgroups:

$$
S_{5}=\bigcup_{\mu \in R_{1}} H_{1} \mu, \quad H=\bigcup_{\tau \in R_{2}} H_{2} \tau, \quad A_{5}=\bigcup_{\nu \in R_{3}} H_{3} v,
$$

where

$$
\begin{aligned}
& H=\langle(23),(24),(25)\rangle \cong S_{4}, \quad H_{1}=\langle(12),(13),(45)\rangle \cong D_{6}, \\
& H_{2}=\langle(23),(45)\rangle \cong V_{4}, \quad H_{3}=\langle(12)(34),(13)(24)\rangle \cong V_{4} \text {, }
\end{aligned}
$$

and $D_{6}$ is the dihedral group of order 12 .

Now we start to prove Theorem 1.2 when $n=5$. Let $\tau=(12) \in S_{5}$. By Theorem 2.5, we see that $K\left(x_{1}, \ldots, x_{5}\right)^{A_{5}}=K\left(F_{1}, \ldots, F_{5}\right)$.

With the aid of a computer, we can evaluate the action of $\tau$ on $K\left(F_{1}, \ldots, F_{5}\right)$ as follows:

$$
\begin{aligned}
& \tau: F_{1} \mapsto a / F_{1}, \quad F_{2} \mapsto F_{3} / F_{1}, \quad F_{3} \mapsto a F_{2} / F_{1}, \\
& F_{4} \mapsto-F_{4}, \quad F_{5} \mapsto-F_{5} \quad \text { when char } K \neq 2 ; \\
& \tau: F_{1} \mapsto a / F_{1}, \quad F_{2} \mapsto F_{3} / F_{1}, \quad F_{3} \mapsto a F_{2} / F_{1}, \\
& F_{4} \mapsto F_{4}+1, \quad F_{5} \mapsto F_{5} \quad \text { when char } K=2 .
\end{aligned}
$$

Case 1: $\operatorname{char} K \neq 2$.

Define

$$
\begin{array}{ll}
G_{1}:=F_{1}, & G_{2}:=F_{4}+1 / F_{4}-1, \quad G_{3}:=F_{4}\left(F_{2}-F_{3} / F_{1}\right), \\
G_{4}:=F_{2}+F_{3} / F_{1}, & G_{5}:=F_{4} F_{5} .
\end{array}
$$


Then we have $K\left(x_{1}, \ldots, x_{5}\right)^{A_{5}}=K\left(F_{1}, \ldots, F_{5}\right)=K\left(G_{1}, \ldots, G_{5}\right)$ and

$$
\tau: G_{1} \mapsto a / G_{1}, \quad G_{2} \mapsto 1 / G_{2}, \quad G_{3} \mapsto G_{3}, \quad G_{4} \mapsto G_{4}, \quad G_{5} \mapsto G_{5} .
$$

So it follows from Theorem 2.1 that $K\left(x_{1}, \ldots, x_{5}\right)^{S_{5}}=K\left(G_{3}, G_{4}, G_{5}\right)\left(G_{1}, G_{2}\right)^{\langle\tau\rangle}$ is rational over $K$.

Case 2: $\operatorname{char} K=2$.

Define

$$
G_{1}:=F_{1}, \quad G_{2}:=F_{2}, \quad G_{3}:=\frac{F_{2} F_{3}}{F_{1}}, \quad G_{4}:=F_{4}+\frac{F_{3}}{F_{1} F_{2}+F_{3}}, \quad G_{5}:=F_{5} .
$$

Then we have $K\left(x_{1}, \ldots, x_{5}\right)^{A_{5}}=K\left(F_{1}, \ldots, F_{5}\right)=K\left(G_{1}, \ldots, G_{5}\right)$ and

$$
\tau: G_{1} \mapsto a / G_{1}, \quad G_{2} \mapsto G_{3} / G_{2}, \quad G_{3} \mapsto G_{3}, \quad G_{4} \mapsto G_{4}, \quad G_{5} \mapsto G_{5} .
$$

We use Theorem 2.1 and find that $K\left(x_{1}, \ldots, x_{5}\right)^{S_{5}}=K\left(G_{3}, G_{4}, G_{5}\right)\left(G_{1}, G_{2}\right)^{\langle\tau\rangle}$ is rational over $K$.

\section{Conic bundles: Another approach when char $K \neq 2$}

Throughout this section we assume that char $K \neq 2$.

In this section, we will give another proof of Theorem 1.2 when $n=3,4$ (and char $K \neq 2)$ by presenting $K\left(x_{1}, \ldots, x_{n}\right)^{S_{n}}$ as the function field of a conic bundle over $\mathbb{P}^{n-1}$.

Consider the action of $S_{n}$ on $K\left(x_{1}, \ldots, x_{n}\right)$ defined by Equation (1-1). Because of Theorem 1.1, we may assume that $a \in K^{\times} \backslash K^{\times 2}$ without loss of generality.

Define $\alpha:=\sqrt{a}$ and $\operatorname{Gal}(K(\alpha) / K)=\langle\rho\rangle$, where $\rho(\alpha)=-\alpha$. Extend the actions of $S_{n}$ and $\rho$ to $K(\alpha)\left(x_{1}, \ldots, x_{n}\right)=K(\alpha) \otimes_{K} K\left(x_{1}, \ldots, x_{n}\right)$ by requiring that $S_{n}$ acts trivially on $K(\alpha)$ and $\tau$ acts trivially on $K\left(x_{1}, \ldots, x_{n}\right)$.

Define $z_{i}:=\left(\alpha-x_{i}\right) /\left(\alpha+x_{i}\right)$ for $1 \leq i \leq n$. We find that $K(\alpha)\left(x_{1}, \ldots, x_{n}\right)=$ $K(\alpha)\left(z_{1}, \ldots, z_{n}\right)$ and

$$
\sigma: z_{i} \mapsto-z_{\sigma(i)}
$$

for any $\sigma \in S_{n} \backslash A_{n}$, and

$$
\rho: \alpha \mapsto-\alpha, \quad z_{i} \mapsto 1 / z_{i}
$$

Define $z_{0}:=z_{1}+\cdots+z_{n}, y_{i}:=z_{i} / z_{0}$ for $1 \leq i \leq n$. Hence $y_{1}+\cdots+y_{n}=1$.

Let $t_{1}, \ldots, t_{n}$ be the elementary symmetric functions of $y_{1}, \ldots, y_{n}$. In particular, $t_{1}=1$. Define $\Delta:=\prod_{1 \leq i<j \leq n}\left(y_{i}-y_{j}\right) \in K\left(y_{1}, \ldots, y_{n}\right)$ and $u:=z_{0} \cdot \Delta$. Note that $\Delta^{2}$ can be written as a polynomial in $t_{1}, \ldots, t_{n}$, and thus in $t_{2}, \ldots, t_{n}$.

Lemma 3.1. $K\left(x_{1}, \ldots, x_{n}\right)^{S_{n}}=K(\alpha)\left(t_{2}, \ldots, t_{n}, u\right)^{\langle\rho\rangle}$ and

$$
\rho: \alpha \mapsto-\alpha, \quad t_{i} \mapsto t_{n-i}\left(t_{n} / t_{n-1}\right)^{i} t_{n}^{-1}, \quad u \mapsto f\left(t_{2}, \ldots, t_{n}\right) \cdot u^{-1},
$$


where $f\left(t_{2}, \ldots, t_{n}\right) \in K\left(t_{2}, \ldots, t_{n}\right)$ is given by

$$
f\left(t_{2}, \ldots, t_{n}\right):=(-1)^{n(n-1) / 2} t_{n}^{-(n-1)}\left(t_{n} / t_{n-1}\right)^{(n+1)(n-2) / 2} \Delta^{2}
$$

and we adopt the convention that $t_{0}=t_{1}=1$.

Proof. Note that $K(\alpha)\left(y_{1}, \ldots, y_{n}, z_{0}\right)=K(\alpha)\left(y_{1}, \ldots, y_{n}, u\right)$. Since $u$ is fixed by the action of $S_{n}$, it follows that $K(\alpha)\left(y_{1}, \ldots, y_{n}, z_{0}\right)^{S_{n}}=K(\alpha)\left(y_{1}, \ldots, y_{n}\right)^{S_{n}}(u)=$ $K(\alpha)\left(t_{2}, \ldots, t_{n}, u\right)$; the last equality follows, for example, from the proof of [Hajja and Kang 1995, Lemma 1] because $\sigma\left(y_{i}\right)=y_{\sigma(i)}$ for any $\sigma \in S_{n}$ and $i$ in $1 \leq i \leq n$.

Thus $K\left(x_{1}, \ldots, x_{n}\right)^{S_{n}}=\left(K(\alpha)^{\langle\rho\rangle}\left(x_{1}, \ldots, x_{n}\right)\right)^{S_{n}}=K(\alpha)\left(x_{1}, \ldots, x_{n}\right)^{\left\langle S_{n}, \rho\right\rangle}=$ $\left(K(\alpha)\left(x_{1}, \ldots, x_{n}\right)^{S_{n}}\right)^{\langle\rho\rangle}=K(\alpha)\left(t_{2}, \ldots, t_{n}, u\right)^{\langle\rho\rangle}$.

It is easy to verify that the action of $\rho$ on $K(\alpha)\left(t_{2}, \ldots, t_{n}, u\right)$ is as stated.

We write $n=2 m+1$ if $n$ is odd, and $n=2 m$ otherwise. Define

$$
u_{i}:=t_{i+1}, \quad u_{n-i}:=\rho\left(t_{i+1}\right)=t_{n-(i+1)} t_{n}^{i} / t_{n-1}^{i+1} \quad \text { for } i=1, \ldots, m-1
$$

and

$$
\begin{cases}u_{m}:=t_{m+1}, \quad u_{m+1}:=\rho\left(t_{m+1}\right)=t_{m} t_{n}^{m} / t_{n-1}^{m+1} & \text { if } n \text { is odd, } \\ u_{m}:=t_{n} / t_{n-1}, & \text { if } n \text { is even. }\end{cases}
$$

Lemma 3.2. $K\left(x_{1}, \ldots, x_{n}\right)^{S_{n}}=K(\alpha)\left(u_{1}, \ldots, u_{n-1}, u\right)^{\langle\rho\rangle}$ and

$$
\begin{aligned}
\rho: \alpha & \mapsto-\alpha, \quad u_{i} \mapsto u_{n-i} \quad \text { for } i=1, \ldots, n-1, \\
u & \mapsto g\left(u_{1}, \ldots, u_{n-1}\right) \cdot u^{-1},
\end{aligned}
$$

where $g\left(u_{1}, \ldots, u_{n-1}\right)=f\left(t_{2}, \ldots, t_{n}\right)$ and $f\left(t_{2}, \ldots, t_{n}\right)$ is given as in $(3-1)$.

Proof. The assertion follows from $K(\alpha)\left(t_{2}, \ldots, t_{n}, u\right)=K(\alpha)\left(u_{1}, \ldots, u_{n-1}, u\right)$ and Lemma 3.1. Indeed we may show $K\left(t_{2}, \ldots, t_{n}\right) \subset K\left(u_{1}, \ldots, u_{n-1}\right)$ as follows. Case 1: $n=2 m+1$ is odd.

The fact that $t_{2}, \ldots, t_{m+1} \in K\left(u_{1}, \ldots, u_{n-1}\right)$ follows from (3-2) and (3-3). We have $t_{n} \in K\left(u_{1}, \ldots, u_{n-1}\right)$ because

$$
\left(\frac{u_{m}^{m+1}}{u_{m-1}^{m}}\right) u_{m+1}^{m}\left(\frac{1}{u_{m+2}}\right)^{m+1}=\left(\frac{t_{m+1}^{m+1}}{t_{m}^{m}}\right)\left(\frac{t_{m} t_{n}^{m}}{t_{n-1}^{m+1}}\right)^{m}\left(\frac{t_{n-1}^{m}}{t_{m+1} t_{n}^{m-1}}\right)^{m+1}=t_{n} .
$$

and $t_{n-1} \in K\left(u_{1}, \ldots, u_{n-1}\right)$ because

$$
t_{n}\left(\frac{u_{m-1}}{u_{m}}\right) u_{m+2}\left(\frac{1}{u_{m+1}}\right)=t_{n}\left(\frac{t_{m}}{t_{m+1}}\right)\left(\frac{t_{m+1} t_{n}^{m-1}}{t_{n-1}^{m}}\right)\left(\frac{t_{n-1}^{m+1}}{t_{m} t_{n}^{m}}\right)=t_{n-1} .
$$

From (3-2) we find that $t_{n-(i+1)}=u_{n-i} t_{n-1}^{i+1} / t_{n}^{i}$ for $1 \leq i \leq m-2$. Thus $t_{m+2}, \ldots, t_{n-2} \in K\left(u_{1}, \ldots, u_{n-1}\right)$.

Case 2: $n=2 m$ is even. That $t_{2}, \ldots, t_{m} \in K\left(u_{1}, \ldots, u_{n-1}\right)$ follows from (3-2). 
From (3-2) and (3-3), we get

$$
\frac{u_{k+1}}{u_{k+2}}=\frac{t_{k}}{t_{k+1}} \cdot \frac{t_{n}}{t_{n-1}}=\frac{t_{k}}{t_{k+1}} \cdot u_{m},
$$

where $k=m, \ldots, 2 m-3$. We find that $t_{k+1}=t_{k} u_{m} u_{k+2} / u_{k+1} \in K\left(u_{1}, \ldots, u_{n-1}\right)$ for $m \leq k \leq 2 m-3$. From (3-2), we have $u_{n-1}=t_{n-2} t_{n} / t_{n-1}^{2}=t_{n-2} u_{m} / t_{n-1}$. Hence $t_{n-1}=t_{n-2} u_{m} / u_{n-1} \in K\left(u_{1}, \ldots, u_{n-1}\right)$.

Since $t_{n}=u_{m} t_{n-1}$, it follows that $t_{n} \in K\left(u_{1}, \ldots, u_{n-1}\right)$.

We will change the variables $u_{1}, \ldots, u_{n-1}$ to $v_{1}, \ldots, v_{n-1}$ as follows. When $n=2 m+1$ is odd, define

$$
v_{i}:=\frac{1}{2}\left(u_{i}+u_{n-i}\right), \quad v_{n-i}:=\frac{1}{2}\left(\alpha\left(u_{i}-u_{n-i}\right)\right) \quad \text { for } i=1, \ldots, m .
$$

When $n=2 m$ is even, define

$v_{m}:=u_{m}, \quad v_{i}:=\frac{1}{2}\left(u_{i}+u_{n-i}\right), \quad v_{n-i}:=\frac{1}{2}\left(\alpha\left(u_{i}-u_{n-i}\right)\right) \quad$ for $i=1, \ldots, m-1$.

Thus $K(\alpha)\left(u_{1}, \ldots, u_{n-1}, u\right)=K(\alpha)\left(v_{1}, \ldots, v_{n-1}, u\right)$.

In these variables, Lemma 3.2 reads as follows:

Lemma 3.3. $K\left(x_{1}, \ldots, x_{n}\right)^{S_{n}}=K(\alpha)\left(v_{1}, \ldots, v_{n-1}, u\right)^{\langle\rho\rangle}$ and

$$
\rho: \alpha \mapsto-\alpha, \quad v_{i} \mapsto v_{i} \quad \text { for } i=1, \ldots, n-1, \quad u \mapsto h\left(v_{1}, \ldots, v_{n-1}\right) \cdot u^{-1},
$$

where $h\left(v_{1}, \ldots, v_{n-1}\right)=f\left(t_{2}, \ldots, t_{n}\right)$ and $f\left(t_{2}, \ldots, t_{n}\right)$ is given as in (3-1).

Hence we get the following theorem, which asserts that $K\left(x_{1}, \ldots, x_{n}\right)^{S_{n}}$ is the function field of a conic bundle over $\mathbb{P}^{n-1}$ of the form $x^{2}-a y^{2}=h\left(v_{1}, \ldots, v_{n-1}\right)$ with affine coordinates $v_{1}, \ldots, v_{n-1}$; see for example [Shafarevich 1974, page 73] for conic bundles over $\mathbb{P}^{1}$.

Theorem 3.4. $K\left(x_{1}, \ldots, x_{n}\right)^{S_{n}}=K\left(x, y, v_{1}, \ldots, v_{n-1}\right)$ and the generators $x, y$, $v_{1}, \ldots, v_{n-1}$ satisfy the relation

$$
x^{2}-a y^{2}=h\left(v_{1}, \ldots, v_{n-1}\right),
$$

where $h\left(v_{1}, \ldots, v_{n-1}\right)=f\left(t_{2}, \ldots, t_{n}\right)$ and $f\left(t_{2}, \ldots, t_{n}\right)$ is given as in (3-1).

Proof. Define

$$
x:=\frac{1}{2}\left(u+\frac{h\left(v_{1}, \ldots, v_{n-1}\right)}{u}\right), \quad y:=\frac{1}{2 \alpha}\left(u-\frac{h\left(v_{1}, \ldots, v_{n-1}\right)}{u}\right) .
$$

Then we get $K\left(x, y, v_{1}, \ldots, v_{n-1}\right) \subset K\left(x_{1}, \ldots, x_{n}\right)^{S_{n}}=K(\alpha)\left(v_{1}, \ldots, v_{n-1}, u\right)$. Thus $K\left(x, y, v_{1}, \ldots, v_{n-1}\right)=K\left(x_{1}, \ldots, x_{n}\right)^{S_{n}}$, since $K\left(x, y, v_{1}, \ldots, v_{n}\right)(u)=$ $K(\alpha)\left(v_{1}, \ldots, v_{n-1}, u\right)$ and $\left[K\left(x, y, v_{1}, \ldots, v_{n}\right)(u): K\left(x, y, v_{1}, \ldots, v_{n}\right)\right]=2$. We also have $x^{2}-a y^{2}=h\left(v_{1}, \ldots, v_{n-1}\right)$ by definition. 
Proof of Theorem 1.2 when $n=3$ and char $K \neq 2$.

Step 1. By Lemma 3.1 we find that $K\left(x_{1}, x_{2}, x_{3}\right)^{S_{3}}=K(\alpha)\left(t_{2}, t_{3}, u\right)^{\langle\rho\rangle}$, where

$$
\rho: \alpha \mapsto-\alpha, \quad t_{2} \mapsto t_{2}^{-2} t_{3}, \quad t_{3} \mapsto t_{2}^{-3} t_{3}^{2}, \quad u \mapsto-t_{2}^{-2} \Delta^{2} \cdot u^{-1} .
$$

Note that $\Delta^{2}=\prod_{1 \leq i<j \leq 3}\left(y_{i}-y_{j}\right)^{2}=t_{2}^{2}-4 t_{2}^{3}-4 t_{3}+18 t_{2} t_{3}-27 t_{3}^{2}$ because $t_{1}=1$.

Define $u_{1}:=t_{2}, u_{2}:=\rho\left(t_{2}\right)=t_{2}^{-2} t_{3}$. Then $K(\alpha)\left(t_{2}, t_{3}, u\right)=K(\alpha)\left(u_{1}, u_{2}, u\right)$ and

$$
\rho: u_{1} \mapsto u_{2} \mapsto u_{1}, \quad u \mapsto g\left(u_{1}, u_{2}\right) \cdot u^{-1},
$$

where $g\left(u_{1}, u_{2}\right)=-1+4 u_{1}+4 u_{2}-18 u_{1} u_{2}+27 u_{1}^{2} u_{2}^{2}$.

Define $v_{1}:=\left(u_{1}+u_{2}\right) / 2$ and $v_{2}:=\alpha\left(u_{1}-u_{2}\right) / 2$. Then $\rho: v_{1} \mapsto v_{1}, v_{2} \mapsto v_{2}$ and $g\left(u_{1}, u_{2}\right)=h\left(v_{1}, v_{2}\right)$, where

$$
h\left(v_{1}, v_{2}\right)=-1+8 v_{1}-18 v_{1}^{2}+27 v_{1}^{4}+(18 / a) v_{2}^{2}-(54 / a) v_{1}^{2} v_{2}^{2}+\left(27 / a^{2}\right) v_{2}^{4} .
$$

Hence $K\left(x_{1}, x_{2}, x_{3}\right)^{S_{3}}=K(\alpha)\left(v_{1}, v_{2}, u\right)^{\langle\rho\rangle}=K\left(x, y, v_{1}, v_{2}\right)$, where

$$
x=\frac{1}{2}\left(u+\frac{h\left(v_{1}, v_{2}\right)}{u}\right), \quad y=\frac{1}{2 \alpha}\left(u-\frac{h\left(v_{1}, v_{2}\right)}{u}\right) .
$$

Note that $x$ and $y$ satisfy the relation

$$
\begin{aligned}
x^{2}-a y^{2} & =h\left(v_{1}, v_{2}\right) \\
& =\left(1+v_{1}\right)\left(-1+3 v_{1}\right)^{3}-(18 / a) v_{2}^{2}\left(-1+3 v_{1}^{2}\right)+\left(27 / a^{2}\right) v_{2}^{4} .
\end{aligned}
$$

Step 2. Suppose that char $K=3$. Then (3-4) becomes $x^{2}-a y^{2}=-1-v_{1}$. Hence $K\left(x_{1}, x_{2}, x_{3}\right)^{S_{3}}=K\left(x, y, v_{1}, v_{2}\right)=K\left(x, y, v_{2}\right)$ is rational over $K$.

Step 3. From now on, we assume that char $K \neq 2,3$.

We normalize the generators $v_{1}$ and $v_{2}$ by defining $T_{1}:=3 v_{1}$ and $T_{2}:=3 v_{2} / a$. We get $K\left(x_{1}, x_{2}, x_{3}\right)^{S_{3}}=K\left(x, y, T_{1}, T_{2}\right)$ with a relation

$$
3 x^{2}-3 a y^{2}=-3+8 T_{1}-6 T_{1}^{2}+T_{1}^{4}+6 a T_{2}^{2}-2 a T_{1}^{2} T_{2}^{2}+a^{2} T_{2}^{4} .
$$

Step 4. We find the singularities of (3-5). We get $x=y=-1+T_{1}=T_{2}=0$. Define $T_{3}:=-1+T_{1}$. The relation (3-5) becomes

$$
3 x^{2}-3 a y^{2}=4 a T_{2}^{2}+a^{2} T_{2}^{4}-4 a T_{2}^{2} T_{3}-2 a T_{2}^{2} T_{3}^{2}+4 T_{3}^{3}+T_{3}^{4} .
$$

Step 5. We blow-up Equation (3-6), that is, define $X_{2}:=x / T_{3}, Y_{2}:=y / T_{3}$ and $T_{4}:=T_{2} / T_{3}$. Then $K\left(x, y, T_{1}, T_{2}\right)=K\left(x, y, T_{2}, T_{3}\right)=K\left(X_{2}, Y_{2}, T_{3}, T_{4}\right)$ and the 
relation (3-6) becomes

$$
\begin{aligned}
3 X_{2}^{2}-3 a Y_{2}^{2} & =4 T_{3}+T_{3}^{2}+4 a T_{4}^{2}-4 a T_{3} T_{4}^{2}-2 a T_{3}^{2} T_{4}^{2}+a^{2} T_{3}^{2} T_{4}^{4} \\
& =\left(T_{3}-a T_{3} T_{4}^{2}\right)^{2}+4\left(T_{3}-a T_{3} T_{4}^{2}\right)+4 a T_{4}^{2} \\
& =\left(T_{3}-a T_{3} T_{4}^{2}\right)\left(4+T_{3}-a T_{3} T_{4}^{2}\right)+4 a T_{4}^{2} .
\end{aligned}
$$

Define

$$
\begin{array}{rlrl}
X_{3} & :=\frac{X_{2}}{T_{3}-a T_{3} T_{4}^{2}}, & Y_{3}:=\frac{Y_{2}}{T_{3}-a T_{3} T_{4}^{2}}, \\
S_{1}:=\frac{4+T_{3}-a T_{3} T_{4}^{2}}{T_{3}-a T_{3} T_{4}^{2}}, & S_{2}:=\frac{T_{4}}{T_{3}-a T_{3} T_{4}^{2}} .
\end{array}
$$

Note that $K\left(X_{2}, Y_{2}, T_{3}, T_{4}\right)=K\left(X_{3}, Y_{3}, S_{1}, S_{2}\right)$. For $S_{1} \in K\left(X_{3}, Y_{3}, S_{1}, S_{2}\right)$, $S_{1}$ is a fractional linear transformation of $T_{3}-a T_{3} T_{4}^{2}$. Hence $T_{3}-a T_{3} T_{4}^{2} \in$ $K\left(X_{3}, Y_{3}, S_{1}, S_{2}\right)$. Thus $T_{4}=S_{2} \cdot\left(T_{3}-a T_{3} T_{4}^{2}\right) \in K\left(X_{3}, Y_{3}, S_{1}, S_{2}\right)$ also. Now $S_{1}$ is a fractional linear transformation of $T_{3}$ with coefficients in $K\left(T_{4}\right)$. Hence $T_{3} \in K\left(X_{3}, Y_{3}, S_{1}, S_{2}\right)$. It follows that $X_{2}, Y_{2} \in K\left(X_{3}, Y_{3}, S_{1}, S_{2}\right)$ also.

The relation (3-7) becomes $3 X_{3}^{2}-3 a Y_{3}^{2}=S_{1}+4 a S_{2}^{2}$, which is linear in $S_{1}$. Hence $K\left(x_{1}, x_{2}, x_{3}\right)^{S_{3}}=K\left(X_{3}, Y_{3}, S_{1}, S_{2}\right)=K\left(X_{3}, Y_{3}, S_{2}\right)$ is rational over $K$.

Step 6. Here is another proof. Instead of the method in Step 5, we may proceed as follows:

Define $X_{4}:=x / T_{3}^{2}, \quad Y_{4}:=y / T_{3}^{2}, \quad T_{4}:=T_{2} / T_{3}$, and $T_{5}:=1 / T_{3}$. Then $K\left(x, y, T_{2}, T_{3}\right)=K\left(X_{4}, Y_{4}, T_{4}, T_{5}\right)$ and (3-6) becomes

$$
3 X_{4}^{2}-3 a Y_{4}^{2}=1-2 a T_{4}^{2}+a^{2} T_{4}^{4}+4 T_{5}-4 a T_{4}^{2} T_{5}+4 a T_{4}^{2} T_{5}^{2} .
$$

The singularities here are $X_{4}=Y_{4}=T_{4} \pm(1 / \sqrt{a})=T_{5}=0$. If we blow-up with respect to $1-a T_{4}^{2}$, that is, define

$$
X_{5}:=X_{4} /\left(1-a T_{4}^{2}\right), \quad Y_{5}:=Y_{4} /\left(1-a T_{4}^{2}\right), \quad T_{6}:=T_{5} /\left(1-a T_{4}^{2}\right),
$$

then $K\left(X_{4}, Y_{4}, T_{4}, T_{5}\right)=K\left(X_{5}, Y_{5}, T_{4}, T_{6}\right)$ and the relation becomes

$$
3 X_{5}^{2}-3 a Y_{5}^{2}=1+4 T_{6}+4 a T_{4}^{2} T_{6}^{2} .
$$

Thus we get $K\left(x_{1}, x_{2}, x_{3}\right)^{S_{3}}=K\left(X_{5}, Y_{5}, T_{4} T_{6}, T_{6}\right)=K\left(X_{5}, Y_{5}, T_{4} T_{6}\right)$ is rational over $K$ because (3-8) becomes linear in $T_{6}$.

Proof of Theorem 1.2 when $n=4$ and char $K \neq 2$.

Step 1. By Lemma 3.1 we find that $K\left(x_{1}, x_{2}, x_{3}, x_{4}\right)^{S_{4}}=K(\alpha)\left(t_{2}, t_{3}, t_{4}, u\right)^{\langle\rho\rangle}$, where

$\rho: \alpha \mapsto-\alpha, \quad t_{2} \mapsto t_{2} t_{3}^{-2} t_{4}, \quad t_{3} \mapsto t_{3}^{-3} t_{4}^{2}, \quad t_{4} \mapsto t_{3}^{-4} t_{4}^{3}, \quad u \mapsto t_{3}^{-5} t_{4}^{2} \Delta^{2} \cdot u^{-1}$, 
where

$$
\begin{aligned}
\Delta^{2}= & \prod_{1 \leq i<j \leq 4}\left(y_{i}-y_{j}\right)^{2} \\
= & t_{2}^{2} t_{3}^{2}-4 t_{2}^{3} t_{3}^{2}-4 t_{3}^{3}+18 t_{2} t_{3}^{3}-27 t_{3}^{4}-4 t_{2}^{3} t_{4}+16 t_{2}^{4} t_{4}+18 t_{2} t_{3} t_{4}-80 t_{2}^{2} t_{3} t_{4} \\
& \quad-6 t_{3}^{2} t_{4}+144 t_{2} t_{3}^{2} t_{4}-27 t_{4}^{2}+144 t_{2} t_{4}^{2}-128 t_{2}^{2} t_{4}^{2}-192 t_{3} t_{4}^{2}+256 t_{4}^{3} .
\end{aligned}
$$

Define $u_{1}:=t_{2}, u_{2}:=t_{4} / t_{3}$ and $u_{3}:=\rho\left(t_{2}\right)=t_{2} t_{4} / t_{3}^{2}$. Then $K(\alpha)\left(t_{2}, t_{3}, t_{4}, u\right)$ $=K(\alpha)\left(u_{1}, u_{2}, u_{3}, u\right)$ and

$$
\rho: \alpha \mapsto-\alpha, \quad u_{1} \mapsto u_{3} \mapsto u_{1}, \quad u_{2} \mapsto u_{2}, \quad u \mapsto g\left(u_{1}, u_{2}, u_{3}\right) \cdot u^{-1},
$$

where

$$
\begin{array}{r}
g\left(u_{1}, u_{2}, u_{3}\right)=\frac{u_{2}}{u_{1} u_{3}}\left(-27 u_{1}^{2} u_{2}^{2}-4 u_{1} u_{2} u_{3}+18 u_{1}^{2} u_{2} u_{3}-6 u_{1} u_{2}^{2} u_{3}+144 u_{1}^{2} u_{2}^{2} u_{3}\right. \\
-192 u_{1} u_{2}^{3} u_{3}+256 u_{1} u_{2}^{4} u_{3}+u_{1}^{2} u_{3}^{2}-4 u_{1}^{3} u_{3}^{2}+18 u_{1} u_{2} u_{3}^{2} \\
\left.-80 u_{1}^{2} u_{2} u_{3}^{2}-27 u_{2}^{2} u_{3}^{2}+144 u_{1} u_{2}^{2} u_{3}^{2}-128 u_{1}^{2} u_{2}^{2} u_{3}^{2}-4 u_{1}^{2} u_{3}^{3}+16 u_{1}^{3} u_{3}^{3}\right) .
\end{array}
$$

Define $v_{1}:=\left(u_{1}+u_{3}\right) / 2, v_{2}:=u_{2}$ and $v_{3}=\alpha\left(u_{1}-u_{3}\right) / 2$. Then we obtain $K(\alpha)\left(u_{1}, u_{2}, u_{3}, u\right)=K(\alpha)\left(v_{1}, v_{2}, v_{3}, u\right)$ and

$$
\rho: \alpha \mapsto-\alpha, \quad v_{1} \mapsto v_{1}, \quad v_{2} \mapsto v_{2}, \quad v_{3} \mapsto v_{3}, \quad u \mapsto h\left(v_{1}, v_{2}, v_{3}\right) \cdot u^{-1},
$$

where $h\left(v_{1}, v_{2}, v_{3}\right)=g\left(u_{1}, u_{2}, u_{3}\right) \in K\left(v_{1}, v_{2}, v_{3}\right)$ is given as

$$
\begin{aligned}
& h\left(v_{1}, v_{2}, v_{3}\right)=\frac{v_{2}}{a v_{1}^{2}-v_{3}^{2}}\left(a v_{1}^{2} v_{2}(-1\right.\left.+4 v_{1}-8 v_{2}\right)^{2}\left(v_{1}^{2}-4 v_{2}+4 v_{1} v_{2}+4 v_{2}^{2}\right) \\
&-2 v_{2} v_{3}^{2}\left(v_{1}^{2}-8 v_{1}^{3}+24 v_{1}^{4}-2 v_{2}+18 v_{1} v_{2}-80 v_{1}^{2} v_{2}\right. \\
&\left.+24 v_{2}^{2}+144 v_{1} v_{2}^{2}-128 v_{1}^{2} v_{2}^{2}-96 v_{2}^{3}+128 v_{2}^{4}\right) \\
&\left.-(1 / a) v_{2} v_{3}^{4}\left(-1+8 v_{1}-48 v_{1}^{2}+80 v_{2}+128 v_{2}^{2}\right)-\left(16 / a^{2}\right) v_{2} v_{3}^{6}\right) .
\end{aligned}
$$

Step 2. Because $h\left(v_{1}, v_{2}, v_{3}\right)$ is still complicated, we define $p, q$ and $r$ as

$$
p:=\frac{1}{2}\left(\frac{1}{u_{1}}+\frac{1}{u_{3}}\right) u_{2}, \quad q:=\frac{\alpha}{2}\left(\frac{1}{u_{1}}-\frac{1}{u_{3}}\right) u_{2}, \quad r:=4 u_{2} .
$$

Then $K(\alpha)\left(v_{1}, v_{2}, v_{3}, u\right)=K(\alpha)(p, q, r, u)$. Indeed we have

$$
\begin{aligned}
& p=\frac{a v_{1} v_{2}}{a v_{1}^{2}-v_{3}^{2}}, \quad q=-\frac{a v_{2} v_{3}}{a v_{1}^{2}-v_{3}^{2}}, \quad r=4 v_{2}, \\
& v_{1}=\frac{a p r}{4\left(a p^{2}-q^{2}\right)}, \quad v_{2}=r / 4, \quad v_{3}=-\frac{a p r}{4\left(a p^{2}-q^{2}\right)} .
\end{aligned}
$$

Hence we obtain $K\left(x_{1}, x_{2}, x_{3}, x_{4}\right)^{S_{4}}=K(\alpha)(p, q, r, u)^{\langle\rho\rangle}$ and

$$
\rho: \alpha \mapsto-\alpha, \quad p \mapsto p, \quad q \mapsto q, \quad r \mapsto r, u \mapsto \frac{r^{2}}{64\left(a p^{2}-q^{2}\right)^{2}} \cdot \frac{H(p, q, r)}{u},
$$


where

$$
\begin{aligned}
H(p, q, r)=a^{2}(p-r+2 p r)^{2}\left(-16 p^{2}+r+4 p r+4 p^{2} r\right) \\
-a\left(-32 p^{2}+r+36 p r-12 p^{2} r-20 r^{2}+72 p r^{2}\right. \\
\left.-96 p^{2} r^{2}-8 r^{3}+32 p^{2} r^{3}\right) q^{2}+16(-1+r)^{3} q^{4} .
\end{aligned}
$$

Define $U:=u \cdot r /\left(8\left(a p^{2}-q^{2}\right)\right)$. Then $K(\alpha)(p, q, r, u)=K(\alpha)(p, q, r, U)$, and $\rho$ acts on $K(\alpha)(p, q, r, U)$ by

$$
\rho: \alpha \mapsto-\alpha, \quad p \mapsto p, \quad q \mapsto q, \quad r \mapsto r, \quad U \mapsto H(p, q, r) / U
$$

Hence $K\left(x_{1}, \ldots, x_{4}\right)^{S_{4}}=K(\alpha)(p, q, r, U)^{\langle\rho\rangle}=K(X, Y, p, q, r)$ where

$$
X=\frac{1}{2}\left(U+\frac{g(p, q, r)}{U}\right), \quad Y=\frac{1}{2 \alpha}\left(U-\frac{g(p, q, r)}{U}\right) .
$$

Note that $X$ and $Y$ satisfy the relation

$$
X^{2}-a Y^{2}=H(p, q, r) .
$$

Step 3. Because $H(p, q, r)$ in (3-9) is a biquadratic equation with respect to $q$ and its constant term has the square factor $(p-r+2 p r)^{2}$, we define $p_{2}:=p-r+2 p r$. Then $p=\left(p_{2}+r\right) /(1+2 r)$. We also define $X_{2}:=X(1+2 r)$ and $Y_{2}:=Y(1+2 r)$. Then $K\left(x_{1}, x_{2}, x_{3}, x_{4}\right)^{S_{4}}=K\left(X_{2}, Y_{2}, p_{2}, q, r\right)$ and (3-10) becomes

$$
\begin{aligned}
& X_{2}^{2}-a Y_{2}^{2}=a^{2} p_{2}^{2}\left(-16 p_{2}^{2}+r-28 p_{2} r+4 p_{2}^{2} r-8 r^{2}+16 p_{2} r^{2}+16 r^{3}\right) \\
&-a\left(-32 p_{2}^{2}+r-28 p_{2} r-12 p_{2}^{2} r-12 r^{2}+120 p_{2} r^{2}\right. \\
&\left.-96 p_{2}^{2} r^{2}+48 r^{3}-48 p_{2} r^{3}+32 p_{2}^{2} r^{3}-64 r^{4}+64 p_{2} r^{4}\right) q^{2} \\
&+16(-1+r)^{3}(1+2 r)^{2} q^{4} .
\end{aligned}
$$

The right hand side is biquadratic in $q$ with constant term on the first line. Hence we define $p_{3}:=p_{2} / q, \quad X_{3}:=X_{2} / q$ and $Y_{3}:=Y_{2} / q$, and the equation becomes quadratic in $q$ :

$$
\begin{aligned}
X_{3}^{2}-a Y_{3}^{2}= & a r(-1+4 r)^{2}\left(-1+a p_{3}^{2}+4 r\right) \\
& +4 a p_{3} r\left(7-7 a p_{3}^{2}-30 r+4 a p_{3}^{2} r+12 r^{2}-16 r^{3}\right) q \\
& +4\left(-1+a p_{3}^{2}-4 r-4 r^{2}\right)\left(4-4 a p_{3}^{2}-12 r+a p_{3}^{2} r+12 r^{2}-4 r^{3}\right) q^{2} .
\end{aligned}
$$

Define $q_{2}:=1 / q, r_{2}:=4 r, X_{4}:=4 X_{3} / q, Y_{4}:=4 Y_{3} / q$. Then

$$
\begin{aligned}
& X_{4}^{2}-a Y_{4}^{2}=4 a r_{2}\left(-1+r_{2}\right)^{2}\left(-1+a p_{3}^{2}+r_{2}\right) q_{2}^{2} \\
& \quad+4 a p_{3} r_{2}\left(28-28 a p_{3}^{2}-30 r_{2}+4 a p_{3}^{2} r_{2}+3 r_{2}^{2}-r_{2}^{3}\right) q_{2} \\
& \quad+\left(-4+4 a p_{3}^{2}-4 r_{2}-r_{2}^{2}\right)\left(64-64 a p_{3}^{2}-48 r_{2}+4 a p_{3}^{2} r_{2}+12 r_{2}^{2}-r_{2}^{3}\right) .
\end{aligned}
$$


Because (3-11) is quadratic in $q_{2}$, we may eliminate a linear term of $q_{2}$ in the usual manner by putting

$$
q_{3}:=2 q_{2}+\frac{p_{3}\left(28-28 a p_{3}^{2}-30 r_{2}+4 a p_{3}^{2} r_{2}+3 r_{2}^{2}-r_{2}^{3}\right)}{\left(-1+r_{2}\right)^{2}\left(-1+a p_{3}^{2}+r_{2}\right)} .
$$

Define

$$
X_{5}:=X_{4}\left(-1+r_{2}\right)\left(-1+a p_{3}^{2}+r_{2}\right), \quad Y_{5}:=Y_{4}\left(-1+r_{2}\right)\left(-1+a p_{3}^{2}+r_{2}\right) .
$$

Then (3-11) becomes

$$
\begin{aligned}
X_{5}^{2}-a Y_{5}^{2}=\left(2+r_{2}\right)^{2}\left(-1+a p_{3}^{2}+r_{2}\right)(4- & \left.4 a p_{3}^{2}-5 r_{2}+r_{2}^{2}\right)^{3} \\
& +a r_{2}\left(-1+r_{2}\right)^{4}\left(-1+a p_{3}^{2}+r_{2}\right)^{3} q_{3}^{2} .
\end{aligned}
$$

Defining

and

$$
q_{4}:=\frac{q_{3}\left(-1+r_{2}\right)^{2}\left(-1+a p_{3}^{2}+r_{2}\right)}{\left(2+r_{2}\right)\left(4-4 a p_{3}^{2}-5 r_{2}+r_{2}^{2}\right)}
$$

$$
X_{6}:=\frac{X_{5}}{\left(2+r_{2}\right)\left(4-4 a p_{3}^{2}-5 r_{2}+r_{2}^{2}\right)}, \quad Y_{6}:=\frac{Y_{5}}{\left(2+r_{2}\right)\left(4-4 a p_{3}^{2}-5 r_{2}+r_{2}^{2}\right)},
$$

we get $K\left(x_{1}, \ldots, x_{4}\right)^{S_{4}}=K\left(X_{6}, Y_{6}, p_{3}, q_{4}, r_{2}\right)$ and the equation becomes

$$
X_{6}^{2}-a Y_{6}^{2}=\left(-1+a p_{3}^{2}+r_{2}\right)\left(\left(4-4 a p_{3}^{2}-5 r_{2}+r_{2}^{2}\right)+a r_{2} q_{4}^{2}\right) .
$$

Step 4. We find the singularities of (3-12). We get $p_{3} \pm(1 / \sqrt{a})=r_{2}=X_{6}=Y_{6}=0$. Blow-up with respect to $-1+a p_{3}^{2}$, that is, define

$$
r_{3}:=r_{2} /\left(-1+a p_{3}^{2}\right), \quad X_{7}:=X_{6} /\left(-1+a p_{3}^{2}\right), \quad Y_{7}:=Y_{6} /\left(-1+a p_{3}^{2}\right) .
$$

Then $K\left(p_{3}, q_{4}, r_{2}, X_{6}, Y_{6}\right)=K\left(p_{3}, q_{4}, r_{3}, X_{7}, Y_{7}\right)$ and (3-12) becomes

$$
X_{7}^{2}-a Y_{7}^{2}=\left(1+r_{3}\right)\left(-4-5 r_{3}+a q_{4}^{2} r_{3}-r_{3}^{2}+a p_{3}^{2} r_{3}^{2}\right) .
$$

Define $p_{4}:=p_{3} r_{3}$. Then

$$
X_{7}^{2}-a Y_{7}^{2}=\left(1+r_{3}\right)\left(-4-5 r_{3}+a q_{4}^{2} r_{3}-r_{3}^{2}+a p_{4}^{2}\right) .
$$

Step 5. Equation (3-13) still has a singular locus $p_{4} \pm q_{4}=r_{3}+1=X_{7}=Y_{7}=0$. If we define $p_{5}:=p_{4}+q_{4}$ and $r_{4}:=r_{3}+1$, it becomes

$$
X_{7}^{2}-a Y_{7}^{2}=r_{4}\left(a p_{5}^{2}-2 a p_{5} q_{4}-3 r_{4}+a q_{4}^{2} r_{4}-r_{4}^{2}\right)
$$

with singular locus $S=\left(p_{5}=r_{4}=X_{7}=Y_{7}=0\right)$. Blowing this up along $S$ by defining $r_{5}:=r_{4} / p_{5}, X_{8}:=X_{7} / p_{5}$, and $Y_{8}:=Y_{7} / p_{5}$, we get

$$
X_{8}^{2}-a Y_{8}^{2}=r_{5}\left(a p_{5}-2 a q_{4}-3 r_{5}+a q_{4}^{2} r_{5}-p_{5} r_{5}^{2}\right) \text {. }
$$


Note that this is linear in $p_{5}$. Hence we conclude that the fixed field $K\left(x_{1}, \ldots, x_{4}\right)^{S_{4}}$ $=K\left(X_{8}, Y_{8}, q_{4}, r_{5}\right)$ is rational over $K$.

\section{Using the structures of rings of invariants}

In this section, we give an another proof of Theorem 1.2 in the case of $n=3,4$ and char $K=2$ by using the structure of $K\left(x_{1}, \ldots, x_{n}\right)^{A_{n}}$. Throughout, we assume that $\operatorname{char} K=2$.

For $1 \leq i \leq n$, let $s_{i}$ be the elementary symmetric function in $x_{1}, \ldots, x_{n}$ of degree $i$.

By Revoy's theorem [1982], the invariant ring $K\left[x_{1}, \ldots, x_{n}\right]^{A_{n}}$ is a free module of rank 2 over the subring $K\left[x_{1}, \ldots, x_{n}\right]^{S_{n}}=K\left[s_{1}, \ldots, s_{n}\right]$. Revoy's theorem is valid for all characteristics. We will find explicitly a free basis of $K\left[x_{1}, \ldots, x_{n}\right]^{A_{n}}$ over $K\left[x_{1}, \ldots, x_{n}\right]^{S_{n}}$ for the case $n=3$, 4. For $n=3$ and $n=4$, it suffices by [Neusel and Smith 2002, Example 1, page 75] to find a polynomial of degree 3 and 6, respectively, that is in $K\left[x_{1}, \ldots, x_{n}\right]^{A_{n}}$ but not in $K\left[x_{1}, \ldots, x_{n}\right]^{S_{n}}$.

Define

$$
\begin{aligned}
& b_{3}:=\sum_{\sigma \in A_{3}} \sigma\left(x_{1} x_{2}^{2}\right)=x_{1} x_{2}^{2}+x_{2} x_{3}^{2}+x_{3} x_{1}^{2}, \\
& b_{4}:=\sum_{\sigma \in A_{4}} \sigma\left(x_{1} x_{2}^{2} x_{3}^{3}\right)= \\
& \begin{aligned}
x_{1}^{2} x_{2}^{3} x_{3}+x_{1}^{3} x_{2} x_{3}^{2}+x_{1} x_{2}^{2} x_{3}^{3}+x_{1}^{3} x_{2}^{2} x_{4}+x_{2}^{3} x_{3}^{2} x_{4}+x_{1}^{2} x_{3}^{3} x_{4} \\
+ \\
+x_{1} x_{2}^{3} x_{4}^{2}+x_{1}^{3} x_{3} x_{4}^{2}+x_{2} x_{3}^{3} x_{4}^{2}+x_{1}^{2} x_{2} x_{4}^{3}+x_{2}^{2} x_{3} x_{4}^{3}+x_{1} x_{3}^{2} x_{4}^{3} .
\end{aligned}
\end{aligned}
$$

For $n=3,4$, it follows that $\left\{1, b_{n}\right\}$ is a free basis of $K\left[x_{1}, \ldots, x_{n}\right]^{A_{n}}$, that is,

$$
\begin{aligned}
K\left[x_{1}, x_{2}, x_{3}\right]^{A_{3}} & =K\left[s_{1}, s_{2}, s_{3}\right] \oplus b_{3} K\left[s_{1}, s_{2}, s_{3}\right], \\
K\left[x_{1}, x_{2}, x_{3}, x_{4}\right]^{A_{4}} & =K\left[s_{1}, s_{2}, s_{3}, s_{4}\right] \oplus b_{4} K\left[s_{1}, s_{2}, s_{3}, s_{4}\right] .
\end{aligned}
$$

We have proved this:

Lemma 4.1. Let $K$ be a field of char $K=2$. Then the fields $K\left(x_{1}, x_{2}, x_{3}\right)^{A_{3}}$ and $K\left(x_{1}, x_{2}, x_{3}, x_{4}\right)^{A_{4}}$ of invariants are given as follows.

(i) $K\left(x_{1}, x_{2}, x_{3}\right)^{A_{3}}=K\left(s_{1}, s_{2}, s_{3}, b_{3}\right)$ with the relation

$$
b_{3}^{2}+b_{3} s_{1} s_{2}+s_{2}^{3}+b_{3} s_{3}+s_{1}^{3} s_{3}+s_{3}^{2}=0 .
$$

(ii) $K\left(x_{1}, x_{2}, x_{3}, x_{4}\right)^{A_{4}}=K\left(s_{1}, s_{2}, s_{3}, s_{4}, b_{4}\right)$ with the relation

$$
b_{4}^{2}+b_{4} s_{1} s_{2} s_{3}+b_{4} s_{3}^{2}+s_{2}^{3} s_{3}^{2}+s_{1}^{3} s_{3}^{3}+s_{3}^{4}+b_{4} s_{1}^{2} s_{4}+s_{1}^{2} s_{2}^{3} s_{4}+s_{1}^{4} s_{4}^{2}=0 .
$$

Proof of Theorem 1.2 when $n=3$ and char $K=2$. First, $\tau$ acts on $K\left(x_{1}, x_{2}, x_{3}\right)^{A_{3}}=$ $K\left(s_{1}, s_{2}, s_{3}, b_{3}\right)$ as

$$
s_{1} \mapsto a s_{2} / s_{3}, \quad s_{2} \mapsto a^{2} s_{1} / s_{3}, \quad s_{3} \mapsto a^{3} / s_{3}, \quad b_{3} \mapsto a^{3} b_{3} / s_{3}^{2} .
$$


Apply Theorem 2.2. We find $K\left(x_{1}, x_{2}, x_{3}\right)^{A_{3}}=K\left(s_{3}, u, v\right)$, where $u$ and $v$ are the same as in Theorem 2.2. It is not difficult to check that

$$
u=\frac{b_{3}+s_{3}}{s_{1}^{2}+s_{2}} \quad \text { and } \quad v=\frac{b_{3}+s_{1} s_{2}}{s_{1}^{2}+s_{2}} .
$$

Moreover, the action of $\tau$ is given by

$$
\tau: s_{3} \mapsto \frac{a^{3}}{s_{3}}, \quad u \mapsto \frac{a u}{u^{2}-u v+v^{2}}, \quad v \mapsto \frac{a v}{u^{2}-u v+v^{2}}
$$

Define $w:=u / v$. Then $K\left(x_{1}, x_{2}, x_{3}\right)^{A_{3}}=K\left(s_{3}, v, w\right)$ and

$$
\tau: s_{3} \mapsto \frac{a^{3}}{s_{3}}, \quad v \mapsto \frac{a}{v\left(1-w+w^{2}\right)}, \quad w \mapsto w .
$$

By Theorem 2.1, $K\left(x_{1}, x_{2}, x_{3}\right)^{S_{3}}=K\left(s_{3}, v, w\right)^{\langle\tau\rangle}$ is rational over $K$.

Proof of Theorem 1.2 when $n=4$ and char $K=2$.

In this case, $\tau$ acts on $K\left(x_{1}, x_{2}, x_{3}, x_{4}\right)^{A_{4}}=K\left(s_{1}, s_{2}, s_{3}, s_{4}, b_{4}\right)$ as

$$
\begin{aligned}
s_{1} & \mapsto a s_{3} / s_{4}, \quad s_{2} \mapsto a^{2} s_{2} / s_{4}, \quad s_{3} \mapsto a^{3} s_{1} / s_{4}, \quad s_{4} \mapsto a^{4} / s_{4}, \\
b_{4} & \mapsto a^{6}\left(b_{4}+s_{1} s_{2} s_{3}+s_{3}^{2}+s_{1}^{2} s_{4}\right) / s_{4}^{3} .
\end{aligned}
$$

Define

$$
t_{1}:=\frac{s_{1} s_{3}}{s_{2}}, \quad t_{2}:=s_{2}, \quad t_{3}:=s_{3}, \quad t_{4}:=\frac{s_{1} s_{2} s_{3}+s_{3}^{2}+s_{1}^{2} s_{4}}{s_{2}^{2}}, \quad t_{5}:=\frac{b_{4}+s_{2}^{3}}{s_{2}} .
$$

It follows that $K\left(s_{1}, s_{2}, s_{3}, s_{4}, b_{4}\right)=K\left(t_{1}, t_{2}, t_{3}, t_{4}, t_{5}\right)$. It is easy to check that the relation among the generators $t_{1}, \ldots, t_{5}$ is given by

$$
t_{1}^{3}+t_{1}^{2} t_{2}+t_{1} t_{2}^{2}+t_{2}^{3}+t_{2} t_{4}^{2}+t_{2} t_{4} t_{5}+t_{2} t_{5}^{2}=0 .
$$

Define

$$
u_{1}:=t_{1}, \quad u_{2}:=\frac{t_{2}}{t_{1}}, \quad u_{3}:=t_{3}, \quad u_{4}:=\frac{t_{4}}{\left(t_{1}+t_{2}\right)}, \quad u_{5}:=\frac{t_{5}}{\left(t_{1}+t_{2}\right)} .
$$

Then we get $K\left(t_{1}, \ldots, t_{5}\right)=K\left(u_{1}, \ldots, u_{5}\right)$ with the relation

$$
u_{2}\left(u_{4}^{2}+u_{4} u_{5}+u_{5}^{2}+1\right)+1=0 .
$$

Because this relation is linear in $u_{2}$, we obtain the following lemma.

Lemma 4.2. $K\left(x_{1}, \ldots, x_{4}\right)^{A_{4}}=K\left(u_{1}, u_{3}, u_{4}, u_{5}\right)$, where

$$
u_{1}=\frac{s_{1} s_{3}}{s_{2}}, \quad u_{3}=s_{3}, \quad u_{4}=\frac{s_{1} s_{2} s_{3}+s_{3}^{2}+s_{1}^{2} s_{4}}{s_{2}\left(s_{2}^{2}+s_{1} s_{3}\right)}, \quad u_{5}=\frac{b_{4}+s_{2}^{3}}{s_{2}\left(s_{2}^{2}+s_{1} s_{3}\right)} .
$$


Now we will prove Theorem 1.2 when $n=4$ and char $K=2$.

Write $p=u_{1}, q=u_{3}, \quad r=u_{4}, \quad s=u_{5}$ and $\tau=(12) \in S_{4} \backslash A_{4}$. Note that $K\left(x_{1}, \ldots, x_{4}\right)^{S_{4}}=K(p, q, r, s)^{\langle\tau\rangle}$ and the action of $\tau$ on $K(p, q, r, s)$ is given by

$$
\begin{aligned}
p & \mapsto \frac{r^{2}+r s+s^{2}+1}{a p}, \\
q & \mapsto \frac{a^{3} p^{6} q}{\left(r^{2}+r s+s^{2}+1\right)^{3}+p^{3} q\left((r+1)\left(r^{2}+r s+s^{2}+1\right)+1\right)}, \\
r & \mapsto r, \quad s \mapsto s+r .
\end{aligned}
$$

Define

$$
t:=\frac{\left(r^{2}+r s+s^{2}+1\right)^{3}}{p^{3} q\left((r+1)\left(r^{2}+r s+s^{2}+1\right)+1\right)} .
$$

Then $K\left(x_{1}, x_{2}, x_{3}, x_{4}\right)^{S_{4}}=K(p, q, r, s)^{\langle\tau\rangle}=K(p, t, r, s)^{\langle\tau\rangle}$ and the action of $\tau$ on $K(p, t, r, s)$ is given by

$$
\tau: p \mapsto\left(r^{2}+r s+s^{2}+1\right) /(a p), \quad t \mapsto t+1, \quad r \mapsto r, \quad s \mapsto s+r .
$$

Define

$$
A:=r+s+r t, \quad B:=(r+s) / s, \quad C:=p r / s .
$$

It follows that $K(p, q, r, s)=K(r, A, B, C)$. Thus we have $K\left(x_{1}, x_{2}, x_{3}, x_{4}\right)^{S_{4}}=$ $K(r)(A, B, C)^{\langle\tau\rangle}$ and

$$
\tau: r \mapsto r, \quad A \mapsto A, \quad B \mapsto \frac{1}{B}, \quad C \mapsto \frac{1}{a}\left(\left(r^{2}+1\right)\left(\frac{1}{B}+B\right)+r^{2}\right) / C .
$$

Apply Theorem 2.1. We find that $K\left(x_{1}, x_{2}, x_{3}, x_{4}\right)^{S_{4}}$ is rational over $K$.

\section{References}

[Hajja 2000] M. Hajja, "Linear and monomial automorphisms of fields of rational functions: Some elementary issues", pp. 137-148 in Algebra and number theory, edited by M. Boulagouaz and J. P. Tignol, Lecture Notes in Pure and Appl. Math. 208, Dekker, New York, 2000. MR 2001b:12009 Zbl 0958.12003

[Hajja and Kang 1995] M. Hajja and M. C. Kang, "Some actions of symmetric groups", J. Algebra 177:2 (1995), 511-535. MR 96i:20013 Zbl 0837.20054

[Hajja and Kang 1997] M. Hajja and M.-C. Kang, "Twisted actions of symmetric groups", J. Algebra 188:2 (1997), 626-647. MR 98b:13003 Zbl 0988.13007

[Iskovskih 1991] V. A. Iskovskih, "Towards the problem of rationality of conic bundles", pp. 50-56 in Algebraic geometry (Chicago, IL, 1989), edited by S. Bloch et al., Lecture Notes in Math. 1479, Springer, Berlin, 1991. MR 93i:14033

[Kang 2001] M.-c. Kang, "The rationality problem of finite group actions", pp. 211-218 in First International Congress of Chinese Mathematicians (Beijing, 1998), AMS/IP Stud. Adv. Math. 20, Amer. Math. Soc., Providence, RI, 2001. MR 2002b:12009

[Kang 2004] M.-C. Kang, "Rationality problem of GL4 group actions", Adv. Math. 181:2 (2004), 321-352. MR 2004k:12008 Zbl 1084.14508 
[Kang 2007] M.-c. Kang, "Some rationality problems revisited", pp. 211-218 in International Congress of Chinese Mathematicians (Hangzhou, 2007), Higher Education Press, Beijing, 2007.

[Kuniyoshi 1955] H. Kuniyoshi, "On a problem of Chevalley”, Nagoya Math. J. 8 (1955), 65-67. MR 16,993d Zbl 0065.02602

[Maeda 1989] T. Maeda, "Noether's problem for A5", J. Algebra 125:2 (1989), 418-430. MR 91c: 12004 Zbl 0697.12018

[Masuda 1955] K. Masuda, "On a problem of Chevalley", Nagoya Math. J. 8 (1955), 59-63. MR $16,993 \mathrm{c}$ Zbl 0065.02601

[Neusel and Smith 2002] M. D. Neusel and L. Smith, Invariant theory of finite groups, Mathematical Surveys and Monographs 94, American Mathematical Society, Providence, RI, 2002. MR 2002k:13012 Zbl 0999.13002

[Revoy 1982] P. Revoy, “Anneau des invariants du groupe alterné”, Bull. Sci. Math. (2) 106:4 (1982), 427-431. MR 84m:13007 Zbl 0507.13009

[Shafarevich 1974] I. R. Shafarevich, Basic algebraic geometry, Die Grundlehren der mathematischen Wissenschaften 213, Springer, New York, 1974. MR 51 \#3163 Zbl 0284.14001

Received September 2, 2009. Revised December 22, 2009.

AKINARI HOSHI

DEPARTMENT OF MATHEMATICS

RIKKYO UNIVERSITY

3-34-1 NISHI-IKEBUKURO

TOSHIMA-KU, TOKYO 171-8501

JAPAN

hoshi@rikkyo.ac.jp

MING-CHANG KANG

DEPARTMENT OF MATHEMATICS

NATIONAL TAIWAN UNIVERSITY

TAIPEI

TAIWAN

kang@math.ntu.edu.tw 


\title{
PACIFIC JOURNAL OF MATHEMATICS
}

\author{
http://www.pjmath.org \\ Founded in 1951 by \\ E. F. Beckenbach (1906-1982) and F. Wolf (1904-1989)
}

\section{EDITORS}

V. S. Varadarajan (Managing Editor)

Department of Mathematics

University of California

Los Angeles, CA 90095-1555

pacific@math.ucla.edu

Vyjayanthi Chari

Department of Mathematics

University of California

Riverside, CA 92521-0135

chari@math.ucr.edu

Robert Finn

Department of Mathematics Stanford University

Stanford, CA 94305-2125

finn@math.stanford.edu

Kefeng Liu

Department of Mathematics

University of California

Los Angeles, CA 90095-1555

liu@math.ucla.edu
Darren Long

Department of Mathematics

University of California

Santa Barbara, CA 93106-3080

long@math.ucsb.edu

Jiang-Hua Lu

Department of Mathematics

The University of Hong Kong

Pokfulam Rd., Hong Kong jhlu@maths.hku.hk

Alexander Merkurjev

Department of Mathematics

University of California

Los Angeles, CA 90095-1555

merkurev@math.ucla.edu
Sorin Popa

Department of Mathematics University of California

Los Angeles, CA 90095-1555 popa@math.ucla.edu

Jie Qing

Department of Mathematics

University of California

Santa Cruz, CA 95064

qing@cats.ucsc.edu

Jonathan Rogawski

Department of Mathematics

University of California

Los Angeles, CA 90095-1555

jonr@math.ucla.edu

\section{PRODUCTION}

pacific@math.berkeley.edu

\begin{abstract}
Silvio Levy, Scientific Editor Matthew Cargo, Senior Production Editor
\end{abstract}
ACADEMIA SINICA, TAIPEI

CALIFORNIA INST. OF TECHNOLOGY

INST. DE MATEMÁTICA PURA E APLICADA

KEIO UNIVERSITY

MATH. SCIENCES RESEARCH INSTITUTE

NEW MEXICO STATE UNIV.

OREGON STATE UNIV.

\section{SUPPORTING INSTITUTIONS}

STANFORD UNIVERSITY
UNIV. OF BRITISH COLUMBIA
UNIV. OF CALIFORNIA, BERKELEY
UNIV. OF CALIFORNIA, DAVIS
UNIV. OF CALIFORNIA, LOS ANGELES
UNIV. OF CALIFORNIA, RIVERSIDE
UNIV. OF CALIFORNIA, SAN DIEGO
UNIV. OF CALIF., SANTA BARBARA

UNIV. OF CALIF., SANTA CRUZ

UNIV. OF MONTANA

UNIV. OF OREGON

UNIV. OF SOUTHERN CALIFORNIA

UNIV. OF UTAH

UNIV. OF WASHINGTON

WASHINGTON STATE UNIVERSITY

These supporting institutions contribute to the cost of publication of this Journal, but they are not owners or publishers and have no responsibility for its contents or policies.

See inside back cover or www.pjmath.org for submission instructions.

The subscription price for 2010 is US \$420/year for the electronic version, and \$485/year for print and electronic.

Subscriptions, requests for back issues from the last three years and changes of subscribers address should be sent to Pacific Journal of Mathematics, P.O. Box 4163, Berkeley, CA 94704-0163, U.S.A. Prior back issues are obtainable from Periodicals Service Company, 11 Main Street, Germantown, NY 12526-5635. The Pacific Journal of Mathematics is indexed by Mathematical Reviews, Zentralblatt MATH, PASCAL CNRS Index, Referativnyi Zhurnal, Current Mathematical Publications and the Science Citation Index.

The Pacific Journal of Mathematics (ISSN 0030-8730) at the University of California, c/o Department of Mathematics, 969 Evans Hall, Berkeley, CA 94720-3840, is published monthly except July and August. Periodical rate postage paid at Berkeley, CA 94704, and additional mailing offices. POSTMASTER: send address changes to Pacific Journal of Mathematics, P.O. Box 4163, Berkeley, CA 94704-0163.

PJM peer review and production are managed by EditFLOW ${ }^{\mathrm{TM}}$ from Mathematical Sciences Publishers.

PUBLISHED BY PACIFIC JOURNAL OF MATHEMATICS

at the University of California, Berkeley 94720-3840

A NON-PROFIT CORPORATION

Typeset in LATEX

Copyright $(\mathrm{C} 2010$ by Pacific Journal of Mathematics 


\section{PACIFIC JOURNAL OF MATHEMATICS}

Volume $248 \quad$ No. $2 \quad$ December 2010

Topological description of Riemannian foliations with dense leaves

Jesús A. ÁlVAREZ LóPEZ and Alberto CANDEL

The nonexistence of quasi-Einstein metrics

JEFFREY S. CASE

Twisted symmetric group actions

AKINARI HoSHI and MING-CHANG KANG

Optimal transportation and monotonic quantities on evolving manifolds

305

HONG HUANG

Hopf structures on the Hopf quiver $Q(\langle g\rangle, g)$

HuA-Lin HuANG, YU Ye and QING ZHaO

Minimal surfaces in $S^{3}$ foliated by circles

Nikolai KuteV and VelichKa Milousheva

Prealternative algebras and prealternative bialgebras

XIANG Ni and CHENGMING BAI

Some remarks about closed convex curves

Ke OU and Shengliang PAN

Orbit correspondences for real reductive dual pairs

SHU-YEN PAN

Graphs of bounded degree and the $p$-harmonic boundary

Michael J. Puls

Invariance of the BFV complex

FLORIAN SCHÄTZ

Some elliptic PDEs on Riemannian manifolds with boundary

YANNICK SIRE and ENRICO VALDINOCI

Representations of Lie superalgebras in prime characteristic, III 